\title{
Using Abstractions for the Verification of Linear Hybrid Systems *
}

\author{
A. Olivero, J. Sifakis and S. Yovine \\ VERIMAG ** \\ Miniparc-Zirst rue Lavoisier \\ 38330 Montbonnot St. Martin, France
}

\section{Introduction}

Hybrid systems are dynamical systems consisting of interacting discrete and continuous components [NSY91, MMP91]. They are used to model the combined behavior of embedded real-time systems and their physical environments. Recently, there have been attempts to develop verification methods for hybrid systems by working in two complementary directions:

- The first direction concerns the identification of subclasses of hybrid systems for which there exist decidability results and effective verification methods for various classes of properties. The main decidability results concern very restricted classes of hybrid systems like timed automata [AD90, ACD90, Alu91, NSY91] and integration graphs [KPSY93].

- The second direction concerns the elaboration of a general verification methodology for classes of systems for which tractable semi-decision methods are applicable. One interesting class is linear hybrid systems [ACHH93, NOSY93], i.e., systems where conditions on discrete transitions and evolution laws of continuous variables are linear constraints.

This paper deals with the verification of linear hybrid systems by bringing results in the two mentioned directions:

- It identifies a class of linear hybrid systems which are shown to be bisimilar to timed automata for which the satisfaction of TCTL properties can be decided by model-checking.

- It studies transformations of general linear hybrid systems inducing abstractions which are timed automata. The properties expressed in $\forall$ TCTL, a fragment of TCTL, are preserved on these systems.

The paper is organized as follows:

Section 2 presents the model of Linear Hybrid Systems (LHS) where continuous variables evolve according to linear functions with slopes varying within an interval. We define two sub-classes of LHS: Constant Slope Hybrid Systems (CSHS) [KPSY93], where the slopes of the evolution functions are constant and $K$-Timed Graphs ( $K$-TG) that are CSHS where for all variables the slope is equal to a constant $K$.

\footnotetext{
* Supported by the ESPRIT project $\mathrm{N}^{\circ} 6021$ REACT-P

$\star$ VERIMAG is a joint laboratory of CNRS, INPG, UJF and VERILOG S.A.
} 
In Sect. 3 we review the syntax and the semantics of the logic TCTL used to describe system properties.

In Sect. 4 we give a simple transformation that maps the sub-class of CSHS with non-zero slopes into equivalent timed graphs in the sense that the underlying models are bisimilar. This implies in particular, that the initial and the transformed system satisfy the same TCTL formulas modulo a linear time transformation. These results are used to show that satisfaction of TCTL is decidable for transformable CSHS.

In Sect. 5 we show that the results of Sect. 4 cannot be extended to general LHS. We propose an extension of the transformation of Sect. 4 which applied to a LHS yields a timed graph which is an abstraction of it. Then we prove that the validity of $\forall$ TCTL formulas is preserved for timed graphs that are abstractions of LHS.

\section{Linear Hybrid Systems}

\subsection{Syntax}

Let $X$ be a set of variables ranging over the set $\mathbb{R}$ of real numbers. A valuation $X_{0}$ of $X$ assigns to each variable $x \in X$ a real number $x_{0} \in \mathbb{R}$. A linear predicate over $X$ is a boolean combination of linear inequalities with integer coefficients over $X$.

A linear hybrid system (LHS) $H$ is a structure $\langle S, X, E, A, B, \phi\rangle$ where:

- $S$ is a finite set of locations. In a graphical representation of the system they are drawn as the nodes of the graph.

- $X$ is a finite set of real-valued variables. These variables change continuously at locations and discretely via transitions.

- $E$ is a finite set of edges. Each edge $e \in E$ is a tuple $\left\langle s, a, \psi, v, s^{\prime}\right\rangle$, where $s \in S$ is the source, $s^{\prime} \in S$ is the target, $a$ is the label, $\psi$ is a linear predicate called the guard, and $v$ is an assignment represented as a set $\left\{x:=v_{x} \mid x \in Y \subseteq X\right\}$ where $v_{x}$ is an interval with end-points in $\mathbb{Z} \cup\{-\infty,+\infty\}$. In the graphical representation, each $e \in E$ is drawn as an edge from the source location to the target location with the label $(a, \psi, v)$.

- $A$ and $B$ associate to each location $s \in S$ the functions $A_{s}$ and $B_{s}$ from $X$ to $\mathbb{Z}$ such that $A_{s} \leq B_{s}$. The rate of change of the variable $x$ at the location $s$ belongs to the closed interval $\left[A_{s}(x), B_{s}(x)\right]$.

- $\phi$ associates with each location $s \in S$ a linear predicate $\phi_{s}$ over $X$ called the invariant at $s$.

A Constant Slope Hybrid System (CSHS) [KPSY93] is a linear hybrid system such that $A=B$. That is, any variable $x$ changes continuously at a constant rate $A_{s}(x)$ at $s$. We denote a CSHS as a tuple $H=\langle S, X, E, A, \phi\rangle$ rather than $H=\langle S, X, E, A, A, \phi\rangle$.

A $K$-Timed Graph ( $K$-TG) is a constant slope hybrid system where all variables are nonnegative and change uniformly at the same rate $K>0$, that is $A_{s}(x)=K$. Guards are boolean combinations of inequalities of the form 
$l \prec x \prec u$ where $\prec \in\{<, \leq\}$ and $l \in \mathbb{N}$ and $u \in \mathbb{N} \cup\{+\infty\}$. We denote a $K$-TG as a tuple $H=\langle S, X, E, \mathbf{K}, \phi\rangle$.

A 1-Timed Graph differs from a timed graph [ACD90, Alu91, NSY91] in the sense that assignments are intervals rather than resets to zero. 1-Timed Graphs are an extension of timed graphs for which all the decidability and modelchecking results concerning timed graphs can also be applied. For this reason 1-timed graphs are hereafter simply called timed graphs.

Notation: If $A$ and $B$ are tuples of scalars and $c$ is a scalar, we write:

- $A(i)$ to represent the $i$-th component of $A$.

- $\frac{c}{A}$ to represent the tuple with $i$-th component $\frac{c}{A(i)}$.

$-A \prec B$ if $A(i) \prec B(i)$ for any component and $\prec \in\{<, \leq\}$.

\subsection{Semantics}

Let $H=\langle S, X, E, A, B, \phi\rangle$ be a linear hybrid system. A state of $H$ is a pair $\left(s, X_{0}\right)$ such that $\phi_{s}\left(X_{0}\right)$. We denote by $Q$ the set of states of $H$. For $q=$ $\left(s, X_{0}\right) \in Q, q(x)$ is the value $x_{0}$ of $x$ at the valuation $X_{0}$.

$H$ changes its state either by a discrete and instantaneous move through an edge, or by a continuous transformation of its variables while time elapses. During the continuous transformations at location $s$ we require that the associated invariant $\phi_{s}$ continuously holds. The semantics of $H$ is a labeled transition system $\langle Q, \rightarrow\rangle$ where the transition relation $\rightarrow$ between states is defined by the following rules:

- An edge $\left\langle s_{0}, a, \psi, v, s_{1}\right\rangle \in E$ is enabled at a state $\left(s_{0}, X_{0}\right)$ if $X_{0}$ satisfies the guard $\psi$. Whenever an edge is enabled at a state it may be taken, and the resulting state is a pair $\left(s_{1}, X_{1}\right)$ where $X_{1}$ is such that for every variable $x \in X, x_{1} \in v_{x}$ if $x:=v_{x} \in v$, and $x_{1}=x_{0}$ otherwise. We denote by $v\left(X_{0}\right)$ the set of such valuations.

$$
\frac{\left\langle s_{0}, a, \psi, v, s_{1}\right\rangle \in E, \psi\left(X_{0}\right), X_{1} \in v\left(X_{0}\right)}{\left(s_{0}, X_{0}\right) \stackrel{a}{\rightarrow}\left(s_{1}, X_{1}\right)}
$$

- Time can progress by $t$ at $\left(s, X_{0}\right)$, if there is a valuation $X_{1}$ reached from $X_{0}$ for a rate $\Lambda \in\left[A_{s}, B_{s}\right]$ and all the intermediate states satisfy the invariant associated to $s$.

$$
\frac{X_{1}=X_{0}+A t, \Lambda \in\left[A_{s}, B_{s}\right], \forall 0 \leq t^{\prime} \leq t . \phi_{s}\left(X_{0}+\Lambda t^{\prime}\right)}{\left(s, X_{0}\right) \stackrel{t}{\rightarrow}\left(s, X_{1}\right)}
$$

We introduce the following definitions:

A trajectory $\sigma$ is an infinite sequence $q_{0} \stackrel{t_{0}}{\mapsto} q_{1} \stackrel{t_{1}}{\mapsto} \ldots$ where for all $i \geq 0 q_{i} \stackrel{t_{i}}{\mapsto} q_{i+1}$ is such that $q_{i} \stackrel{t_{i}}{\rightarrow} q_{i+1}$, or there exists a label $a$ such that $q_{i} \stackrel{a}{\rightarrow} q_{i+1}$ and $t_{i}=0$. A position $\pi$ of $\sigma$ is a pair $(i, t)$ with $0 \leq t \leq t_{i}$. We define a total order $\preceq$ on the positions, as $(i, t) \preceq\left(j, t^{\prime}\right)$ iff $i<j$ or $i=j \wedge t<t^{\prime}$. Let $q_{i}=\left(s_{i}, X_{0 i}\right)$ and $q_{i+1}=\left(s_{i}, X_{0 i+1}\right)$ where $X_{0 i+1}=X_{0 i}+A_{i} \cdot t_{i}$, with $A_{i} \in\left[A_{s_{i}}, B_{s_{i}}\right]$. The state at position $(i, t)$ of $\sigma$ is $\sigma(i, t)=\left(s_{i}, X_{0 i}+A_{i} . t\right)$. The time elapsed at position 
$(i, t)$ is $\tau_{\sigma}(i, t)=t+\sum_{j<i} t_{j}$, that is, the time elapsed since the beginning of the trajectory. A trajectory $\sigma$ diverges if for all $t \in \mathbb{R}^{+}$there exists a position $\pi$ such that $\tau_{\sigma}(\pi)>t$. The set of all divergent trajectories is represented by $\Sigma$, and the set of all divergent trajectories starting at $q$ by $\Sigma(q)$.

Remark. A different semantics for LHS is proposed in [AHH93] for which the results developed in the paper are also valid.

Example 1. The temperature of a tank grows linearly with time at rates belonging to the interval $[4,6]$. For safety reasons the temperature must be kept within a minimum of 79 and a maximum of 90 degrees. A controller cools the tank by moving two independent rods. The rates of cooling belong to the intervals $[-4,-1]$ and $[-3,-2]$, respectively. Furthermore, each rod can be used again only if a given time has elapsed since the end of its previous movement. These minimum times are 7 and 5 minutes, respectively. If the temperature cannot be decreased because there is no available rod, a complete shutdown is required. A variant of this example is given in [JLHM91, NOSY93]. Figure 1 shows a linear hybrid system for the controller.

We use three variables: $\theta$ measures the temperature of the tank and $x_{1}$ and $x_{2}$ count the time elapsed since the last use of the respective rod. Initially, the temperature takes its minimum value and both rods can be used.

The equations at locations specify the evolution laws and the predicate specifies the invariant for the location.

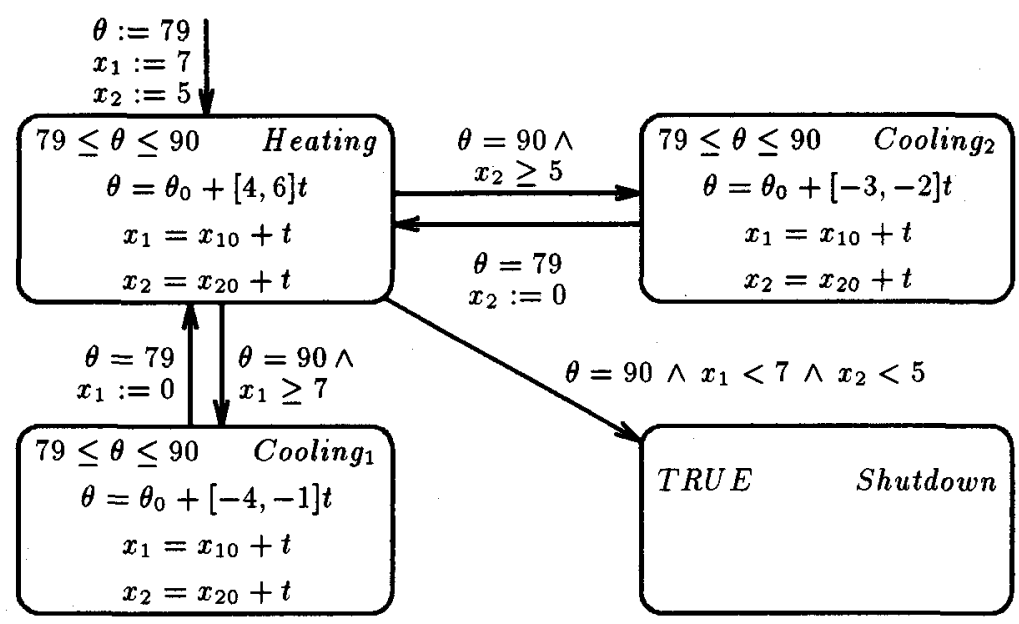

Fig. 1. Temperature control system 


\section{The Logic TCTL}

The real-time temporal logic TCTL is a real-time extension of CTL [CES86]. Many versions of TCTL have been defined [ACD90, Alu91, HNSY92]. We adopt here the one defined in [ACD90] using the temporal operators $\exists \mathcal{U}$ and $\forall \mathcal{U}$ of CTL with subscripts that limit their scope in time.

Intuitively, $f_{1} \exists \mathcal{U}_{\# n} f_{2}$ means that there exists a trajectory such that $f_{2}$ holds at some state at a time $t$ satisfying $t \# n$ and $f_{1}$ continuously holds at all intermediate states. $f_{1} \forall \mathcal{U}_{\# n} f_{2}$ means that for every trajectory the above property holds. For example, we can write true $\exists \mathcal{U}_{<5} p$ to say that along some trajectory, $p$ becomes true before time 5 .

\subsection{Syntax}

Let $P$ be a finite set of atomic propositions. The formulas of TCTL are defined by the following grammar:

$$
f::=p|l \prec x \prec u| \neg f\left|f_{1} \vee f_{2}\right| f_{1} \exists \mathcal{U}_{\# n} f_{2} \mid f_{1} \forall \mathcal{U}_{\# n} f_{2}
$$

where $p \in P, x \in X, l, u \in \mathbb{Z} \cup\{-\infty,+\infty\}, \prec \in\{<, \leq\}, n \in \mathbb{N}$ and $\# \in\{<, \leq$ $,>, \geq,=\}$.

We use standard abbreviations such as $\forall \diamond_{\# n} f$ for true $\forall \mathcal{U}_{\# n} f, \exists \diamond_{\# n} f$ for true $\exists \mathcal{U}_{\# n} f, \exists \square_{\# n} f$ for $\neg \forall \diamond_{\# n} \neg f$ and $\forall \square_{\# n} f$ for $\neg \exists \diamond_{\# n} \neg f$. In Sect. 5 we use the abbreviation $f_{1} \forall \mathcal{W}_{\# n} f_{2}$ for the formula $\neg\left(\neg f_{1} \exists \mathcal{U}_{\# n} \neg f_{2}\right)$. The unrestricted temporal operators of CTL correspond to TCTL operators subscripted by $\geq 0$.

\subsection{Semantics}

TCTL formulas are interpreted over the divergent trajectories generated by the model of a linear hybrid system. Let $H$ be a LHS, $\langle Q, \rightarrow\rangle$ its model, and $M$ an interpretation of atomic propositions over the set of states, i.e. $M(p) \subseteq Q$ for all $p \in P$.

The satisfaction relation the state $q \in Q$ satisfies the formula $f$ under the interpretation $M$, denoted $q \vDash_{M} f$, is inductively defined as follows:

$q \vDash_{M} p$ iff $q \in M(p)$;

$q \models_{M} l \prec x \prec u$ iff $l \prec q(x) \prec u$;

$q \models_{M} \neg f$ iff $q \not \nvdash_{M} f$;

$q \vDash_{M} f_{1} \vee f_{2}$ iff $q \vDash_{M} f_{1}$ or $q \vDash_{M} f_{2}$;

$q \models_{M} f_{1} \exists \mathcal{U}_{\# n} f_{2}$ iff for some sequence $\sigma \in \Sigma(q)$ there exists a position $\pi$ such that $\sigma(\pi) \vDash_{M} f_{2}$ and $\tau_{\sigma}(\pi) \# n$, and for all $\pi^{\prime} \preceq \pi, \sigma\left(\pi^{\prime}\right) \vDash_{M} f_{1} \vee f_{2} \wedge$ $\tau_{\sigma}\left(\pi^{\prime}\right) \# n$;

$q \vDash_{M} f_{1} \forall \mathcal{U}_{\# n} f_{2}$ iff for all sequences $\sigma \in \Sigma(q)$ there exists a position $\pi$ such that $\sigma(\pi) \vDash_{M} f_{2}$ and $\tau_{\sigma}(\pi) \# n$, and for all $\pi^{\prime} \preceq \pi, \sigma\left(\pi^{\prime}\right) \vDash_{M} f_{1} \vee f_{2} \wedge \tau_{\sigma}\left(\pi^{\prime}\right) \# n$;

$H$ satisfies $f$ under $M$, denoted $H \models_{M} f$, if all the states of $H$ satisfy $f$.

Example 2. We express in TCTL three properties of Example 1: 
- The location Shutdown is not reachable:

$$
\text { (Heating } \left.\wedge \theta=79 \wedge x_{1}=7 \wedge x_{2}=5\right) \Rightarrow \forall \square \neg \text { Shutdown }
$$

- Rod 1 (Rod 2) can be used again only if at least 7 minutes ( 5 minutes) have elapsed since the end of its previous movement:

$$
\begin{aligned}
& \text { (Heating } \left.\wedge \theta=79 \wedge x_{1}=0\right) \Rightarrow \neg \exists \diamond_{<7} \text { Cooling }_{1} \\
& \text { (Heating } \left.\wedge \theta=79 \wedge x_{2}=0\right) \Rightarrow \neg \exists \text { Cooling }_{2}
\end{aligned}
$$

\section{From Constant Slope Hybrid Systems to Timed Graphs}

We define the class of Non-Zero Constant Slope Hybrid System, denoted CSHS $\neq 0$, such that:

- All the rates are different from zero.

- The invariant and guards are boolean combinations of inequalities of the form $l \prec x \prec u$ with $\prec \in\{<, \leq\}$.

- For all edges $e=\left\langle s, a, \psi, v, s^{\prime}\right\rangle \in E$ and for all variables $x \in X$, if the rates of change of $x$ are different at $s$ and $s^{\prime}$, then $x:=v_{x}$ is in $v$.

- At any location $s$ any variable with positive (negative) slope has a finite lower (upper) bound in the invariant $\phi_{s}$.

In this section we show how a given $\operatorname{CSHS}_{\neq 0}$ can be transformed into an equivalent TG. To this end, we use two transformations: the $K$-transformation and the $\frac{1}{K}$-transformation, whose successive application to a $\mathrm{CSHS}_{\neq 0}$ gives a $K$-timed graph and a timed graph. The key idea is to apply linear transformations of variables which are proper to each location. For the $K$-transformation the aim is to obtain the same evolution laws for all variables and locations by multiplying by appropriate constants. Consequently, conditions and assignments are modified. The $\frac{1}{K}$-transformation consists in changing the time scale. Both transformations preserve bisimilarity of the underlying models.

\subsection{Translating CSHS $_{\neq 0}$ into $K$-TG}

The $\boldsymbol{K}$-transformation. Given $H=\langle S, X, E, A, \phi\rangle$ in CSHS $_{\neq 0}$, we define the set of functions $\kappa=\left\{\kappa_{s} \mid s \in S\right\}$ as follows:

$$
\kappa_{s}(X)=\frac{K}{A_{s}} X+C_{s}
$$

where:

- $K=\operatorname{lcm}\left\{\operatorname{abs}\left(A_{s}(x)\right) \mid s \in S, x \in X\right\}$, that is, $K$ is the least common multiple of the absolute values of the rates appearing in $H$, and

- $C_{s}$ is a tuple such that $C_{s}(x)$ is the maximum of the absolute values of all the constants appearing in assignments to $x$ in edges with target location $s$ or in conditions on $x$ in guards of edges with source location $s$, multiplied by $\operatorname{abs}\left(\frac{K}{A_{s}(x)}\right)$. 
For given $H=\langle S, X, E, A, \phi\rangle$ in $\mathrm{CSHS}_{\neq 0}$, we construct a $K$-timed graph $H_{K}=$ $\left\langle S, X^{\prime}, E^{\prime}, \mathbf{K}, \phi^{\prime}\right\rangle$ such that:

- $X^{\prime}$ is in bijection with $X$.

- For each $e=\left\langle s, a, \psi, v, s^{\prime}\right\rangle \in E$ we construct the edge $e^{\prime}=\left\langle s, a, \psi^{\prime}, v^{\prime}, s^{\prime}\right\rangle \in$ $E^{\prime}$ where $\psi^{\prime}$ is obtained replacing each condition of the form $l \prec x \prec u$ appearing in $\psi$ by a condition $l^{\prime}=\frac{K}{A_{s}(x)} l+C_{s}(x) \prec x^{\prime} \prec \frac{K}{A_{s}(x)} u+C_{s}(x)=u^{\prime}$ when the rate $A_{s}(x)$ is positive, or $u^{\prime} \prec x^{\prime} \prec l^{\prime}$ otherwise. The assignment $v^{\prime}$ is obtained replacing each $x:=[\alpha, \beta] \in v$ by $x^{\prime}:=\left[\frac{K}{A_{s}(x)} \alpha+C_{s}(x), \frac{K}{A_{s}(x)} \beta+\right.$ $\left.C_{s}(x)\right]=\left[\alpha^{\prime}, \beta^{\prime}\right]$ when the rate $A_{s}(x)$ is positive, or $x^{\prime}:=\left[\beta^{\prime}, \alpha^{\prime}\right]$ otherwise.

$-\phi_{s}^{\prime}$ is obtained from $\phi_{s}$ exactly as $\psi^{\prime}$ is obtained from $\psi$.

Remark. When the slope of $x$ is positive (negative) and $l$ or $\alpha(u$ or $\beta$ ) are $-\infty(\infty)$ we take the conjunction with the invariant $\phi_{s}$ before applying the transformation. This operation does not change the semantics of the system.

Notice that $H_{K}$ is constructed from $H$ so as the relation $X^{\prime}=\kappa_{s}(X)$ holds at each location $s$.

Property Preservation by $\boldsymbol{K}$-transformation. We want to prove that $H$ and $H_{K}$ are bisimilar. We recall here the definition of bisimulation.

Definition 1. Let $T_{1}=\left\langle Q_{1}, \rightarrow_{1}\right\rangle$ and $T_{2}=\left\langle Q_{2}, \rightarrow_{2}\right\rangle$ be two labeled transition systems over a set $\mathcal{L}$ of labels and a relation $\rho \subseteq Q_{1} \times Q_{2}$.

The relation $\rho$ is a bisimulation between $T_{1}$ and $T_{2}$, if $\rho\left(q_{1}, q_{2}\right)$ implies for every label $\ell \in \mathcal{L}$ :

- If $q_{1} \stackrel{\ell}{\rightarrow} 1 q_{1}^{\prime}$, then there exists $q_{2}^{\prime}$ such that $q_{2} \stackrel{\ell}{\rightarrow} 2 q_{2}^{\prime}$ and $\rho\left(q_{1}^{\prime}, q_{2}^{\prime}\right)$.

- If $q_{2} \stackrel{\ell}{\rightarrow} 2 q_{2}^{\prime}$, then there exists $q_{1}^{\prime}$ such that $q_{1} \stackrel{\ell}{\rightarrow} 1 q_{1}^{\prime}$ and $\rho\left(q_{1}^{\prime}, q_{2}^{\prime}\right)$.

We say that $T_{1}$ and $T_{2}$ are bisimilar, denoted $T_{1} \sim T_{2}$, if there exists a bisimulation $\rho$ between $T_{1}$ and $T_{2}$.

For $H_{1}$ and $H_{2}$ linear hybrid systems, we write $\left\langle H_{1}, q_{1}\right\rangle \sim\left\langle H_{2}, q_{2}\right\rangle$ if the transition systems generated respectively from $q_{1}$ and $q_{2}$ are bisimilar. We write $H_{1} \sim H_{2}$ if there exists a bisimulation $\rho$ between the models of $H_{1}$ and $H_{2}$.

We naturally extend the function $\kappa$ to a relation on states in the following way:

$$
\kappa\left((s, X),\left(s^{\prime}, X^{\prime}\right)\right) \text { iff } s=s^{\prime} \text { and } X^{\prime}=\kappa_{s}(X)
$$

Proposition 2. For all $\mathrm{CSHS}_{\neq 0} H, H \sim H_{K}$.

Proof. It is straightforward to prove that the relation $\kappa$ is a bisimulation between $H$ and $H_{K}$.

Our goal is to prove that $H$ and $H_{K}$ satisfy the same TCTL formulas modulo the transformation of the predicates over $X$ according to $\kappa$. So, for any formula $f$ we define a formula $|f|_{K}$, such that $H$ satisfies $f$ iff $H_{K}$ satisfies $|f|_{K}$. The formula $|f|_{K}$ is obtained by substitution of any predicate of the form $l \prec x \prec u$ ocurring in $f$ by $\bigvee_{s \in S} p_{s} \wedge l^{\prime} \prec x^{\prime} \prec u^{\prime}$, where $p_{s}$ is an atomic proposition which holds only at location $s$ and $l^{\prime} \prec x^{\prime} \prec u^{\prime}$ is the condition defined in Sect. 4.1. 
Proposition 3. For any $H$ in $C S H S_{\neq 0}$ and for any TCTL formula $f$ :

$$
H \vDash_{M} f \text { iff } H_{K} \vDash_{M}|f|_{K}
$$

Proof. By induction on the structure of the formula based on the fact that $H \sim H_{K}$.

Example (continued). We consider the CSHS $H$ obtained from the LHS of Fig. 1 by taking the rate of $\theta$ equal to 6 at location Heating, to -4 at location Cooling and to -3 at location Cooling 2 . The evolution laws for $\theta$ are different at all locations. However, since all conditions are of the form $\theta=c$ (for a constant $c$ ) we can add the assignment $\theta:=c$ without changing the semantics of the system. Thus, $H \in$ CSHS $_{\neq 0}$ and the $K$-transformation is applicable to obtain the $K$-TG of Fig. 2. The set of functions $\kappa$ is given by:

$$
\begin{aligned}
& \theta^{\prime}=2 \theta+180 \text { at location Heating } \\
& \theta^{\prime}=-3 \theta+270 \text { at location Cooling } \\
& \theta^{\prime}=-4 \theta+360 \text { at location Cooling } \\
& x_{1}^{\prime}=12 x_{1}+84 \text { at all locations } \\
& x_{2}^{\prime}=12 x_{2}+60 \text { at all locations }
\end{aligned}
$$

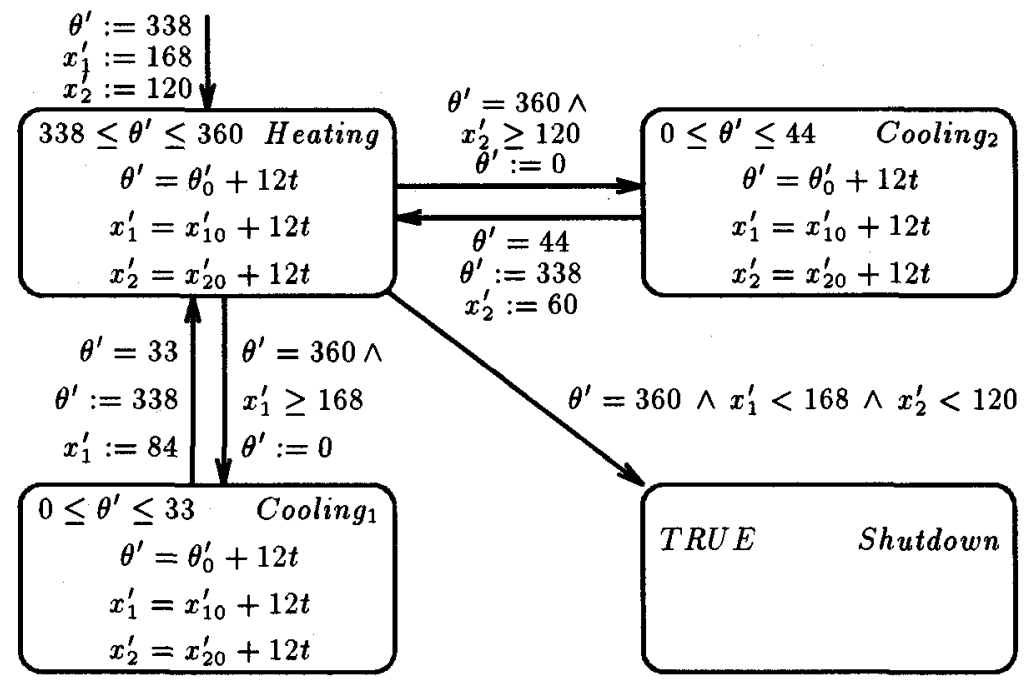

Fig. 2. Temperature control system ( $K-\mathrm{TG})$

The transformation of formulas (1), (2) and (3) according to $|\cdots|_{K}$ gives:

$$
\begin{aligned}
\left(\text { Heating } \wedge \theta^{\prime}=338 \wedge x_{1}^{\prime}=168 \wedge x_{2}^{\prime}=120\right) & \Rightarrow \forall \square \neg \text { Shutdown } \\
\left(\text { Heating } \wedge \theta^{\prime}=338 \wedge x_{1}^{\prime}=84\right) & \Rightarrow \neg \exists \diamond_{<7} \text { Cooling }_{1} \\
\left(\text { Heating } \wedge \theta^{\prime}=338 \wedge x_{2}^{\prime}=60\right) & \Rightarrow \neg \exists \diamond_{<5} \text { Cooling }_{2}
\end{aligned}
$$


By Proposition 3, $H$ satisfies formulas (1), (2) and (3) iff $H_{K}$ satisfies formulas (4), (5) and (6), respectively.

\subsection{Translating $K$-TG into TG}

The problem of verifying TCTL formulas on $K$-timed graphs can be reduced to the problem of verifying TCTL formulas on timed graphs. Given a $K$-timed graph $H_{K}$ a timed graph $H_{1}$ can be obtained such that the satisfaction of a TCTL formula $f$ on $H_{K}$ is equivalent to the satisfaction of a formula $|f|_{\frac{1}{K}}$ obtained by transforming $f$.

The $\frac{1}{K}$-transformation. Given a $K$-timed graph $H_{K}=\langle S, X, E, \mathbf{K}, \phi\rangle$, this transformation gives a timed graph $H_{1}=\langle S, X, E, \mathbf{1}, \phi\rangle$. That is, an evolution law of the form $x=x_{0}+K t$ in $H_{K}$ is replaced by the law $x=x_{0}+t$ in $H_{1}$.

Property Preservation by $\frac{1}{K}$-transformation. Given a formula $f$, we define a formula $|f|_{\frac{1}{K}}$ by replacing the subscripts $\# n$ by \#Kn in the temporal operators.

Proposition 4. For any $K$-timed graph $H_{K}$ and any TCTL formula $f$ :

$$
H_{K} \vDash_{M} f \text { iff } H_{1} \vDash_{M}|f|_{\frac{1}{K}}
$$

Example (continued). The $\frac{1}{K}$-transformation is applicable to the system $H_{K}$ of Fig. 2 to obtain a TG $H_{1}$. The transformation of formulas (4), (5) and (6) according to $|\cdots|_{\frac{1}{K}}$ gives:

$$
\begin{aligned}
\text { (Heating } \left.\wedge \theta^{\prime}=338 \wedge x_{1}^{\prime}=168 \wedge x_{2}^{\prime}=120\right) & \Rightarrow \forall \square \neg \text { Shutdown } \\
\left(\text { Heating } \wedge \theta^{\prime}=338 \wedge x_{1}^{\prime}=84\right) & \Rightarrow \neg \exists \diamond_{<84} \text { Cooling }_{1} \\
\left(\text { Heating } \wedge \theta^{\prime}=338 \wedge x_{2}^{\prime}=60\right) & \Rightarrow \neg \exists \diamond_{<60} \text { Cooling }_{2}
\end{aligned}
$$

By Proposition $4 H_{K}$ satisfies formulas (4), (5) and (6) iff $H_{1}$ satisfies formulas (7), (8), and (9), respectively.

The following results were obtained using the tool Krovos [NSY92, OY92] to verify the formulas (7), (8) and (9) on the timed graph $H_{1}$.

\begin{tabular}{||l|r|r|r||}
\hline formula & time in sec. & iterations & result \\
\hline$(7)$ & 0.050 & 4 & true \\
\hline$(8)$ & 0.066 & 4 & true \\
\hline$(9)$ & 0.083 & 4 & true \\
\hline
\end{tabular}

Then $H_{K}$ satisfies formulas (4), (5) and (6) and by Proposition 3 the $\operatorname{CSHS}_{\neq 0}$ $H$ satisfies formulas (1), (2) and (3). 
Synthesis of the results. By combination of Propositions 3 and 4, we obtain:

Proposition 5. For any $C S H S_{\neq 0} H$ and any TCTL formula f:

$$
H \vDash_{M} f \text { iff } H_{K} \vDash_{M}|f|_{K} \text { iff } H_{1} \vDash_{M} \|\left.\left. f\right|_{K}\right|_{\frac{1}{K}}
$$

This proposition shows that the model-checking problem for $\mathrm{CSHS}_{\neq 0}$ and TCTL is reduced to the problem of model-checking for TG. Now, the model-checking algorithm in [HNSY92] can be adapted for the timed graphs considered here, that is timed graphs having assignments of intervals instead of resets to zero. It can be shown that in this case the meaning of the formulas can be expressed with the same class of state predicates.

\section{From Linear Hybrid Systems to Timed Graphs}

In this section we extend the translation method proposed for CSHS to LHS. We first define a subclass of LHS, denoted LHS $\neq 0$, such that:

- For all locations $s \in S$ and variables $x \in X$, the end-points of the rate interval $A_{s}(x)$ and $B_{s}(x)$ are both positive or both negative, that is $0 \notin$ $\left[A_{s}(x), B_{s}(x)\right]$.

- The invariant and the guards are boolean combinations of inequalities of the form $l \prec x \prec u$ with $\prec \in\{<, \leq\}$.

- For all edges $e \in E$ and for all variables $x \in X$, if the rates of change of $x$ are different at the source location and at the target location, then $x$ must be in the assignment.

- At any location $s$ any variable with positive (negative) slope has a finite lower (upper) bound in the invariant $\phi_{s}$.

\subsection{Translating LHS $_{\neq 0}$ into $K$-TG}

The following proposition shows that unfortunately the result that for any $H$ in $\mathrm{CSHS}_{\neq 0}$ there exist a bisimilar $K-\mathrm{TG}$ cannot be extended to $\mathrm{LHS}_{\neq 0}$.

Proposition 6. Given a $H=\langle S, X, E, A, B, \phi\rangle$ in $L H S_{\neq 0}$ such that for some $s \in S$

$-A_{s}<B_{s}$

- there exists $e \in E$ such that $e=\left\langle s, a,[L, U], v, s^{\prime}\right\rangle$

there exists no $H_{K}$ in $K-T G$ with initial state $q_{K}$ such that $\langle H, q\rangle \sim\left\langle H_{K}, q_{K}\right\rangle$ for all the states $q$ from which there exists a trajectory crossing the edge $e$.

The Generalized $\boldsymbol{K}$-transformation. Proposition 6 states that it is not possible (in general) to transform a given LHS $_{\neq 0}$ into a bisimilar $K$-TG. However, we define a transformation of $H$ into an abstract $H^{\prime}$, in the sense that every transition in the model of $H$ corresponds to a transition in the model of $H^{\prime}$ (the model of $H$ simulates the model of $H^{\prime}$ ). 
Given $H=\langle S, X, E, A, B, \phi\rangle$ in LHS $_{\neq 0}$, and a set of variables $X^{\prime}$ in bijection with $X$, we define the relation $\kappa$ as the set $\kappa=\left\{\kappa_{s} \mid s \in S\right\}$ relating $X$ and $X^{\prime}$. as follows:

$$
\kappa_{s}\left(X, X^{\prime}\right) \text { iff } \frac{K}{B_{s}} X+C_{s} \leq X^{\prime} \leq \frac{K}{A_{s}} X+C_{s}
$$

where:

$-K=\operatorname{lcm}\left\{\operatorname{abs}\left(A_{s}(x)\right), \operatorname{abs}\left(B_{s}(x)\right) \mid x \in X, s \in S\right\}$, that is $K$ is the least common multiple of the absolute values of all different end-points of the rate intervals.

- $C_{s}$ is a tuple such that $C_{s}(x)$ is the maximum of the absolute values of all the constants appearing in assignments to $x$ in edges with target location $s$ or in conditions on $x$ in guards of edges with source location $s$, multiplied by $\max \left(\operatorname{abs}\left(\frac{K}{A_{s}(x)}\right), \operatorname{abs}\left(\frac{K}{B_{s}(x)}\right)\right)$.

From $H$ and $\kappa$ the $K$-timed graph $H_{K}=\left\langle S, X^{\prime}, E^{\prime}, \mathbf{K}, \phi^{\prime}\right\rangle$ is defined as follows:

- $X^{\prime}$ is in bijection with $X$.

- For each $e=\left\langle s, a, \psi, v, s^{\prime}\right\rangle \in E$ we construct the edge $e^{\prime}=\left\langle s, a, \psi^{\prime}, v^{\prime}, s^{\prime}\right\rangle \in$ $E^{\prime}$ where $\psi^{\prime}$ is obtained replacing each condition of the form $l \prec x \prec u$ appearing in $\psi$ by a condition $l^{\prime}=\frac{K}{B_{s}(x)} l+C_{s}(x) \prec x^{\prime} \prec \frac{K}{A_{s}(x)} u+C_{s}(x)=u^{\prime}$ when $A_{s}(x)$ and $B_{s}(x)$ are positive, or $u^{\prime} \prec x^{\prime} \prec l^{\prime}$ otherwise. The assignment $v^{\prime}$ is obtained replacing each $x:=[\alpha, \beta] \in v$ by $x^{\prime}:=\left[\frac{K}{B_{s}(x)} \alpha+\right.$ $\left.C_{s}(x), \frac{K}{A_{s}(x)} \beta+C_{s}(x)\right]=\left[\alpha^{\prime}, \beta^{\prime}\right]$ when $A_{s}(x)$ and $B_{s}(x)$ are positive, or $x^{\prime}:=\left[\beta^{\prime}, \alpha^{\prime}\right]$ otherwise.

- $\phi_{s}^{\prime}$ is obtained from $\phi_{s}$ exactly as $\psi^{\prime}$ is obtained from $\psi$.

The remark of Sect. 4.1 is also applicable in this case. Is important to note that when $A=B, H$ is a CSHS $\neq 0$ and the generalized $K$-transformation coincides with the $K$-transformation defined in Sect. 4.1.

Simulation. We want to prove that $H$ simulates $H_{K}$. We first recall the definition of simulation.

Definition 7. Let $T_{1}=\left\langle Q_{1}, \rightarrow_{1}\right\rangle$ and $T_{2}=\left\langle Q_{2}, \rightarrow_{2}\right\rangle$ be two labeled transition systems over a set $\mathcal{L}$ of labels and a relation $\rho \subseteq Q_{1} \times Q_{2}$.

The relation $\rho$ is a simulation from $T_{1}$ to $T_{2}$, if $\rho\left(q_{1}, q_{2}\right)$ implies for every label $\ell \in \mathcal{L}$ :

- If $q_{1} \stackrel{\ell}{\rightarrow} 1 q_{1}^{\prime}$, then there exists $q_{2}^{\prime}$ such that $q_{2} \stackrel{\ell}{\rightarrow} 2 q_{2}^{\prime}$ and $\rho\left(q_{1}^{\prime}, q_{2}^{\prime}\right)$.

We say that $T_{1}$ simulates $T_{2}$, denoted $T_{1} \sqsubseteq T_{2}$, if there exists a relation $\rho$ such that $\rho$ is a simulation from $T_{1}$ to $T_{2}$.

For $H_{1}$ and $H_{2}$ linear hybrid systems, we write $H_{1} \sqsubseteq H_{2}$ if there exists a simulation $\rho$ from the model of $H_{1}$ to the model of $H_{2}$.

We naturally extend the relation $\kappa$ on states in the following way:

$$
\kappa\left((s, X),\left(s^{\prime}, X^{\prime}\right)\right) \text { iff } s=s^{\prime} \text { and } \kappa_{s}\left(X, X^{\prime}\right)
$$

Lemma 8. For all $L H S_{\neq 0} H, H \sim_{\kappa} H_{K}$ if only timed transitions are considered. 
Lemma 9. For all $L H S_{\neq 0} H, H \sqsubseteq H_{K}$ if only discrete transitions are considered.

Proposition 10. For all $L H S_{\neq 0} H, H \sqsubseteq H_{K}$.

Proof. By combining Lemmas 8 and 9.

Property Preservation by $\boldsymbol{K}$-transformation. We show that an interesting subset of TCTL, called $\forall$ TCTL, is preserved by the relation $K$ from $H_{K}$ to $H$, that is, if $f \in \forall$ TCTL is satisfied by $H_{K}$ then $H$ satisfies a corresponding formula | $\left.f\right|_{K} \in \forall$ TCTL. $\forall T C T L$ is the subset of TCTL defined as follows:

$$
f::=p|\neg p| l \prec x \prec u\left|f_{1} \wedge f_{2}\right| f_{1} \vee f_{2}\left|f_{1} \forall \mathcal{W}_{\# n} f_{2}\right| f_{1} \forall \mathcal{U}_{\prec n} f_{2}
$$

where $f_{1} \forall \mathcal{W}_{\# n} f_{2}$ and $p, x, l, u, \prec, n$ and \# are as in definition of Sect. 3.1.

For any formula $f$ in $\forall$ TCTL the formula $|f|_{K}$ is obtained by replacing any predicate of the form $l \prec x \prec u$ by $\frac{l-C_{s}(x)}{K} A_{s}(x) \prec x^{\prime} \prec \frac{u-C_{s}(x)}{K} B_{s}(x)$.

Proposition 11. For any $L H S_{\not 0} H$ and any $\forall T C T L$ formula $f$,

$$
\text { if } H_{K} \models_{M} f \text { then } H \vDash_{M}|f|_{K} \text {. }
$$

Example 3. Consider the example of Sect. 1 modeled by the LHS $H$ of Fig. 1. The relation $\kappa$ for $H$ is:

$$
\begin{aligned}
2 \theta+270 \leq \theta^{\prime} & \leq 3 \theta+270 \quad \text { at location Heating } \\
-12 \theta+1080 \leq \theta^{\prime} \leq-3 \theta+1080 & \text { at location Cooling } \\
-6 \theta+540 \leq \theta^{\prime} \leq-4 \theta+540 & \text { at location Cooling } \\
& x_{1}^{\prime}=12 x_{1}+84 \quad \text { at all locations } \\
& x_{2}^{\prime}=12 x_{2}+60 \quad \text { at all locations }
\end{aligned}
$$

Figure 3 shows the $K$-TG $H_{K}$ obtained. The $\frac{1}{K}$-transformation is applicable to the system $H_{K}$ to obtain a TG $H_{1}$. Consider the following formulas:

$$
\begin{aligned}
\text { (Heating } & \left.\wedge 428 \leq \theta^{\prime} \leq 507 \wedge x_{1}^{\prime}=168 \wedge x_{2}^{\prime}=120\right) \Rightarrow \forall \square \neg \text { Shutdown }(10) \\
& \left(\text { Heating } \wedge 428 \leq \theta^{\prime} \leq 507 \wedge x_{1}^{\prime}=84\right) \Rightarrow \neg \exists \diamond_{<84} \text { Cooling }_{1}(11) \\
& \left(\text { Heating } \wedge 428 \leq \theta^{\prime} \leq 507 \wedge x_{2}^{\prime}=60\right) \Rightarrow \neg \exists \diamond_{<60} \text { Cooling }_{2}(12)
\end{aligned}
$$

It can be shown that if $H_{1}$ satisfies formulas (10), (11) and (12) then the LHS $\neq 0$ $H$ of Fig. 1 satisfies formulas (1), (2) and (3).

The following results were obtained using the tool Kronos to verify the formulas (10), (11) and (12) on the TG $H_{1}$.

\begin{tabular}{||l|r|r|r||}
\hline formulatime in sec. & iterations & result \\
\hline$(10)$ & 0.084 & 7 & false \\
\hline$(11)$ & 0.117 & 4 & true \\
\hline$(12)$ & 0.084 & 4 & true \\
\hline
\end{tabular}

Notice that formulas (11) and (12) are verified which implies that formulas (2) and (3) are satisfied by the system of Fig. 1. On the contrary, formula (10) is not verified and nothing can be said about the validity of formula (1). 


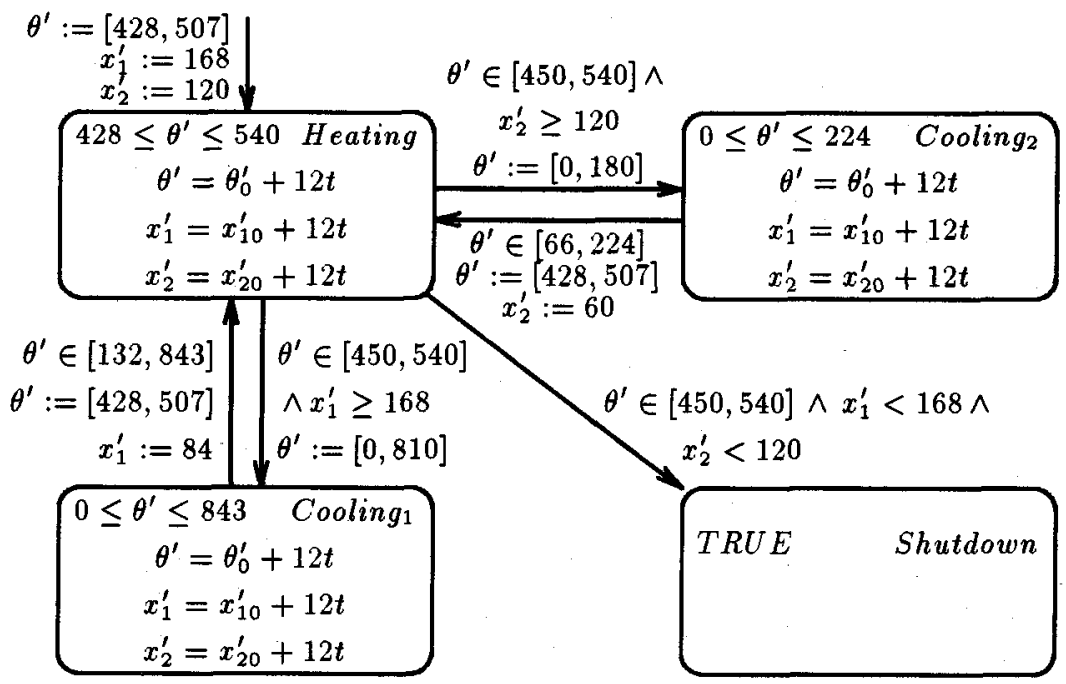

Fig. 3. Temperature control system $\left(H_{K}\right)$

\section{Conclusion}

As mentioned in the introduction, the paper contributes in two different directions to the development of a verification methodology for LHS:

- it shows that the model checking method for TCTL on timed graphs can be extended to a non-trivial class of CSHS, the so called Non-Zero Constant Slope Hybrid Systems CSHS $\neq 0$. Even though the latter class is semantically equivalent to timed graphs, we noticed that several examples of LHS in the literature happen to belong to $\mathrm{CSHS}_{\neq 0}$.

- it shows that well-known results of property preservation by abstractions can be adapted to the case of $\mathrm{LHS}_{\neq 0}$. The generalized $K$-transformation presented in Sect. 5 maps this class to timed graphs and, consequently, the verification of a large class of properties such as invariance and bounded inevitability, on some element of LHS $_{\neq 0}$, can be reduced to the verification of the same property on the corresponding timed graph. It remains to evaluate through examples the usefulness of this approach, that is how much faithful are the abstractions defined by $K$.

These results should be related to the results of [PV94] which consider almost the same class of LHS by using two different approaches. The restriction to have assignments for variables whose evolution law changes is essential for both. The differences concern our requirement that at some position the sign of the rate of the variables cannot change while we accept also open intervals for guards and assignments. The restriction to closed intervals is essential for the decidability result presented in [PV94]. This result is certainly an important one and stronger than our results if they are restricted to decidability of the reachability problem. However, a general comparison is difficult for at least two reasons. The result 
in [PV94] is obtained by digitization which means that it is hardly exploitable in practice and it concerns only reachability properties; it is not obvious that it can be extended to other properties, for instance inevitability properties.

\section{References}

[ACD90] R. Alur, C. Courcoubetis, and D. Dill. Model-checking for real-time systems. In Proc. 5th LICS, pages 414-425. IEEE Comp. Soc. Press, 1990.

[ACHH93] R. Alur, C. Courcoubetis, T. A. Henzinger, and Pei-Hsin Ho. Hybrid automata: an algorithmic approach to the specification and analysis of hybrid systems. In Workshop on Theory of Hybrid Systems, Lyngby, Denmark, June 1993. LNCS 736, Springer-Verlag.

[AD90] R. Alur and D. Dill. Automata for modeling real-time systems. In Proc. 17th ICALP, pages 322-335. LNCS 443, Springer-Verlag, 1990.

[AHH93] R. Alur, T. Henzinger, and P.-H. Ho. Automatic symbolic verification of embedded systems. In 14 th Annual Real-Time Systems Symposium, pages 2-11. IEEE Comp. Soc. Press, 1993.

[Alu91] R. Alur. Techniques for automatic verification of real-time systems. $\mathrm{PhD}$ thesis, Department of Computer Science, Stanford University, August 1991.

[CES86] E.M. Clarke, E.A. Emerson, and A.P. Sistla. Automatic verification of finite-state concurrent systems using temporal logic specifications. ACM Transactions on Programming Languages and Systems, 8(2):244-263, 1986.

[HNSY92] T. Henzinger, X. Nicollin, J. Sifakis, and S. Yovine. Symbolic modelchecking for real-time systems. In Proc. 7th LICS. IEEE Comp. Soc. Press, 1992.

[JLHM91] M. Jaffe, N. Leveson, M. Heimdahl, and B. Melhart. Software requirements analysis for real-time process-control systems. IEEE Transactions on Software Engineering, 17(3):241-258, 1991.

[KPSY93] Y. Kesten, A. Pnueli, J. Sifakis, and S. Yovine. Integration graphs: a class of decidable hybrid systems. In Workshop on Theory of Hybrid Systems, Lyngby, Denmark, June 1993. LNCS 736, Springer-Verlag.

[MMP91] O. Maler, Z. Manna, and A. Pnueli. From timed to hybrid systems. In Proc. REX Workshop "Real-Time: Theory in Practice", the Netherlands, June 1991. LNCS 600, Springer-Verlag.

[NOSY93] X. Nicollin, A: Olivero, J. Sifakis, and S. Yovine. An approach to the description and analysis of hybrid systems. In Workshop on Theory of Hybrid Systems, Lyngby, Denmark, June 1993. LNCS 736, Springer-Verlag.

[NSY91] X. Nicollin, J. Sifakis, and S. Yovine. From ATP to timed graphs and hybrid systems. In Proc. REX Workshop "Real-Time: Theory in Practice", the Netherlands, June 1991. LNCS 600, Springer-Verlag.

[NSY92] X. Nicollin, J. Sifakis, and S. Yovine. Compiling real-time specifications into extended automata. IEEE TSE Special Issue on Real-Time Systems, 18(9):794-804, September 1992.

[OY92] A. Olivero and S. Yovine. Kronos: a Tool for Verifying Real-time Systems. User's Guide and Reference Manual. VERIMAG, Grenoble, France, 1992.

[PV94] A. Puri and P. Varaiya. Decidability of hybrid systems with rectangular differential inclusions. This volume. 